\title{
Direct Laser Lithography and Its Applications
}

\author{
Hyug-Gyo Rhee \\ Korea Research Institute of Standards and Science \\ Republic of Korea
}

\section{Introduction}

Computer Generated Hologram (CGH) is widely used for testing of large aspheric surfaces. It, however, is difficult to fabricate by the well-known E-beam methods because most of the CGH requires a large diameter $(\varnothing 75$ to $1,000 \mathrm{~mm}$ ) and a tough precision (position accuracy of each line in the CGH should be less than $50 \mathrm{~nm}$.). In this case, the direct laser lithography can be a proper choice to fabricate the CGH becuse it can easily extend the size (patterned area) with high precision.

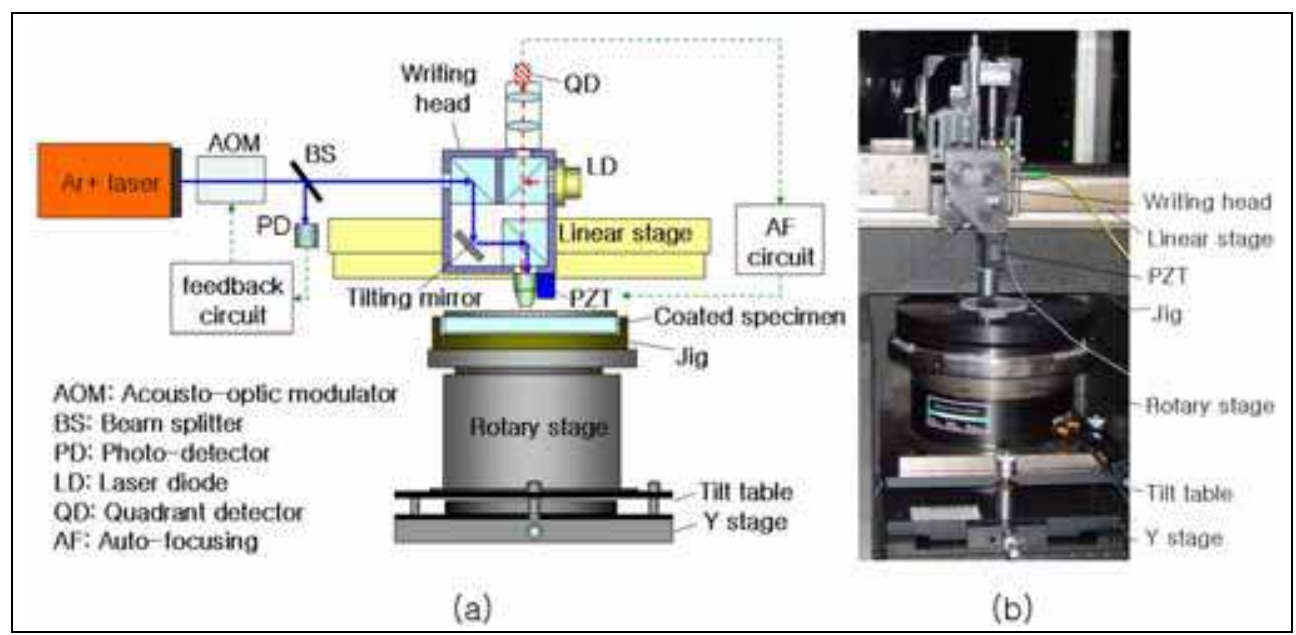

Fig. 1. (a) Configuration and (b) photographic view of the direct laser lithographic system.

Figure 1 (a) shows the configuration of typical direct laser lithographic system, which includes (1) the intensity stabilization and control part, (2) the writing head with autofocusing mechanism, and (3) the moving part. The photographic view of the assembled lithographic system is shown in Fig. 1 (b). The blue light in this figure is the lithographic beam whose wavelength is $457.9 \mathrm{~nm}$. The laser lithographic system requires a high stability of the intensity of the source. In the fluctuating spectrum of a gaseous laser, large variations may be found in the low frequency range, from dc to several hundred $\mathrm{Hz}$, and considerably smaller variations in the frequency band to several hundred $\mathrm{kHz}$. The first is attributed to such main factors as thermal variations of the resonant cavity, mechanical vibrations, dust Source: Lithography, Book edited by: Michael Wang,

ISBN 978-953-307-064-3, pp. 656, February 2010, INTECH, Croatia, downloaded from SCIYO.COM 
particles and air currents, instability, and the hum of the power supply. The second is mainly due to the oscillations in the plasma of the discharge column, especially in the region of the space charge at the cathode. The Ar+ laser that is used as a microfabrication source in our system also shows the above-mentioned beam fluctuations. For the source stabilization, we have introduced an Acousto-Optic Modulator (AOM), a photodetector, and produced a servo controller for controlling the AOM modulation depth.

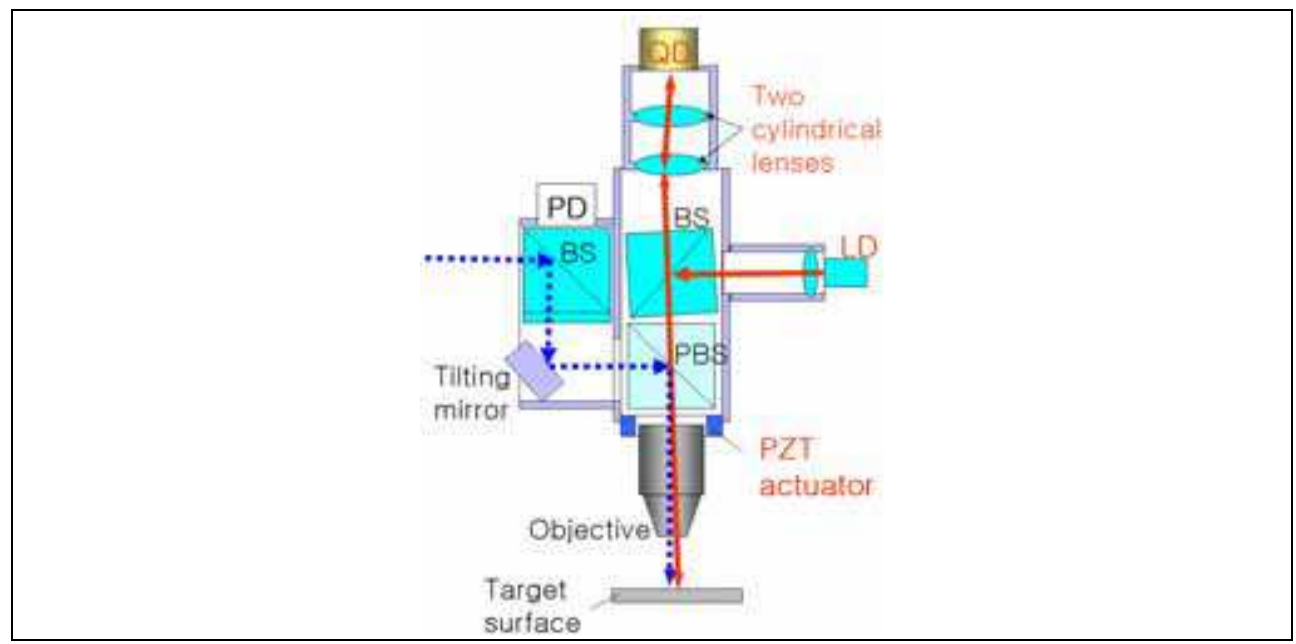

Fig. 2. Configuration of the writing head.

The stabilized lithographic beam from the AOM comes into the writing head as shown in Fig. 2. The dotted line and the solid line represent the lithographic and the autofocusing beam, respectively. The tilting mirror permits a direction change of the lithographic beam with a $0.02^{\circ}$ resolution in order to compensate for the run-out error of the rotary motor. One of important functions of the writing head is autofocusing. Furthermore, 20X, 50X, and 100X objectives are available in our system to alter the lithographic spot size. Each objective requires a different set of astigmatic lenses for the best autofocusing performance. Table 1 shows the specifications of the moving part. A laser interferometer was also placed in the system to check the exact position of the writing head.

\begin{tabular}{|c|c|c|}
\hline Specification & Rotary stage & Linear stage \\
\hline Range & $360^{\circ}$ & $200 \mathrm{~mm}$ \\
\hline Control type & Closed loop & Closed loop \\
\hline Feedback sensor & Rotary encoder & Linear encoder \\
\hline Resolution & 0.0547 sec. & $10 \mathrm{~nm}$ \\
\hline Max. Speed & $600 \mathrm{rpm}$ & $100 \mathrm{~mm} / \mathrm{s}$ \\
\hline Axis loads & Wafer chuck $+1 \mathrm{~kg}$ & $7 \mathrm{~kg}$ \\
\hline
\end{tabular}

Table 1. Specification of the rotary and the linear stage.

Figure 3 shows the main page of the operating software we developed. A pattern for fabrication is displayed in Part 1. Part 2 is a stage motion test panel. Part 3 shows the signals 
from the motor encoders, the laser interferometer, the PD of the intensity control part, and the status of the shutter. The overall fabrication state is displayed in Part 4.

The details of the intensity stabilization and the autofocusing are presented in Section 2. In Section 3, some applications are described.

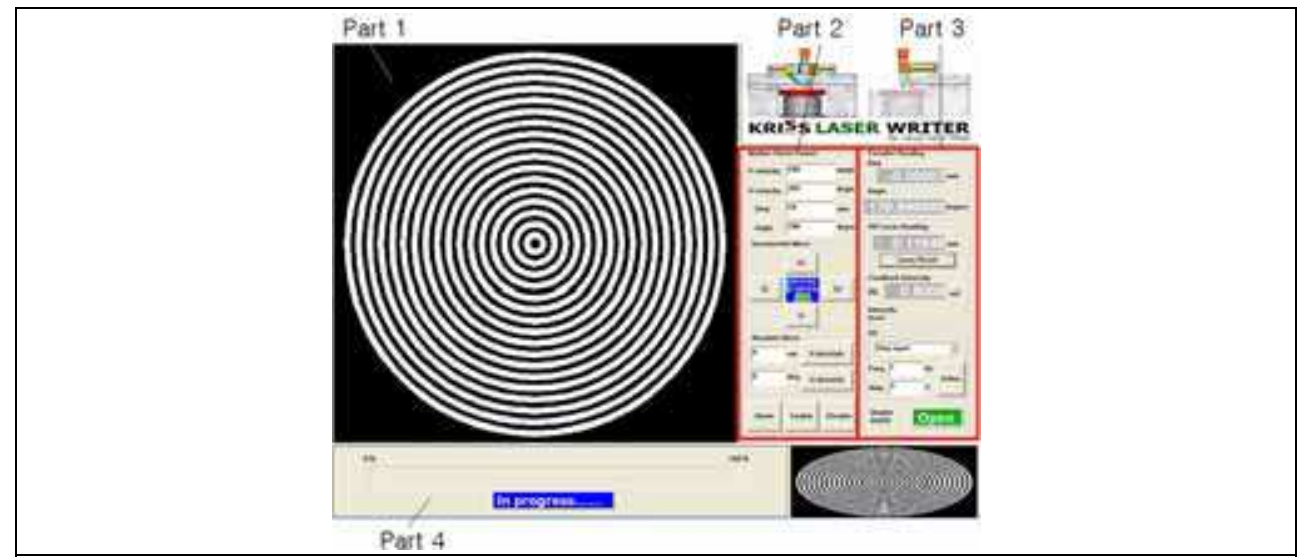

Fig. 3. Operating software.

\section{Key technologies}

\subsection{Laser power stabilization}

The source beam is the Ar+ laser with and output power of $1.5 \mathrm{~W}$ at $514.5 \mathrm{~nm}$ and of 300 $\mathrm{mW}$ at $457.9 \mathrm{~nm}$ wavelength. Its beam property is linearly polarized owing to plasma tube ends with Brewster angle cut. This is an important factor for power stabilization with our system. The stabilization system consists of the AOM, a cube beam splitter (BS), the photodetector (PD), and the control servo circuit. The description of each part is as follow: The $\mathrm{AOM}$ is installed in the direction of propagation of the beam to conduct active power control, so that it is independent of the laser system used. For this reason, this setup will be able to apply to different kind of laser. The power control scheme using the AOM is that if its modulation depth is changed to minimize the intensity fluctuation in real time, the constant output power can be obtained. Hear the allowable modulation voltage limit corresponding to the modulation depth is up to $1 \mathrm{~V}$. Therefore, to be well operated the $\mathrm{AOM}$, the voltage between $0 \mathrm{~V}$ and $1 \mathrm{~V}$ have to be introduced to the AOM-RF driver. If the input voltage exceeds the limitation, the AOM loss their function as the active power controller. The first order beam passing through the AOM is split into two parts by BS. A small portion of reflected beam (about $8 \%$ ) is measured by the PD for stabilization itself. A linear polarizer (LP) is employed in front of the PD to prevent bring about serious damage to the PD. The used PD has the damage threshold of $100 \mathrm{~mW}$ for continuous wave and 0.5 $\mathrm{J} / \mathrm{cm}^{2}$ for $10 \mathrm{~ns}$ pulse, respectively. We carried out our experiment with the power of 65 $\mathrm{mW}$. The servo controller was designed with an upper unity gain at $10 \mathrm{kHz}$ to achieve high gain at low frequencies. The gain at frequencies below $100 \mathrm{~Hz}$ was at least $60 \mathrm{~dB}$, which was sufficient gain to reduce the main fluctuation noise of around $100 \mathrm{~Hz}$.

The mechanism of the stabilization part is that the detected photocurrent by PD is converted to voltage and then is compared with extremely low noise voltage reference (Analogue 
Devices AD581JH) in the servo controller. An error signal out of the controller is introduced into the AOM-RF driver to change the diffractive ratio of the AOM instantaneously. Also owing to time taken to circulate through the loop is very short (about $1 \mu \mathrm{s}$ ), continuous stabilization is achieved instantly. In this way, the first order beam power is maintained constantly. In our experiment, the AOM and the controller have $20 \mathrm{MHz}$ and $5 \mathrm{MHz}$ bandwidth, respectively. These are enough values that cover the noise frequency band of the used $\mathrm{Ar}+$ laser.

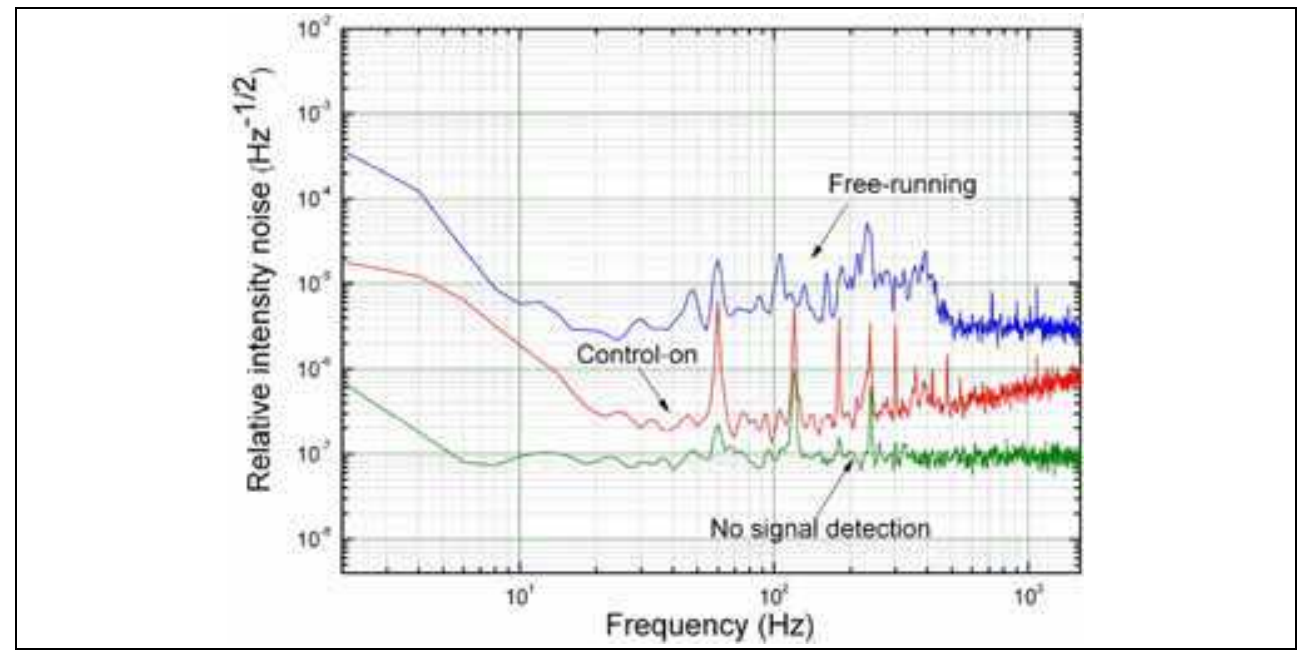

Fig. 4. Relative intensity noise of the Ar+ laser. The free-running mode shows the main noise frequency band of the laser.

Figure 4 shows relative intensity noise as measured by a FFT spectrum analyzer with DC to $102.4 \mathrm{kHz}$ bandwidth (Stanford Research SR785) according to three different conditions which are free-running, control-on, and no signal detection. The free-running mode shows the large fluctuating noise at the low frequency range, from $20 \mathrm{~Hz}$ to $500 \mathrm{~Hz}$. Once the control loop is on, we can see the noise level is dramatically reduced. If we look around the relative intensity noise level at relatively large fluctuation frequency of $100 \mathrm{~Hz}$, we can see the value of $1.1 \times 10^{-5} \mathrm{~Hz}^{-1 / 2}$ is reduced to $2.1 \times 10^{-7} \mathrm{~Hz}^{-1 / 2}$ by about two orders. This result shows our stabilization system is able to carry out a function as a power controller. In the Fig. 4, there are regular bursts with $60 \mathrm{~Hz}$ spacing at the control-on or the no signal detection node. The reason is due to the florescent light incident on the PD. Later we could see that these bursts disappeared on the screen of the FFT spectrum analyzer as soon as the light was off. And if we expand the frequency band in the FFT spectrum analyzer, we can confirm our stabilization part has the noise control band up to $4 \mathrm{kHz}$. This range is sufficient to control the output power of various gaseous lasers including the Ar+ laser.

The performance of the stabilization part is shown in Fig. 5. It shows clearly the difference of the power stabilities before and after operating the control loop. Long term stability obtained by the control loop is $\pm 0.20 \%$ for 12 minutes. This is a considerably reduced quantity comparable with the free-running mode of $\pm 0.77 \%$. Normally, it takes 11 or 12 hours to complete a piece of CGH. So we observed the long term stability for over 10 hours but the stability shown in the above result was not nearly changed. 


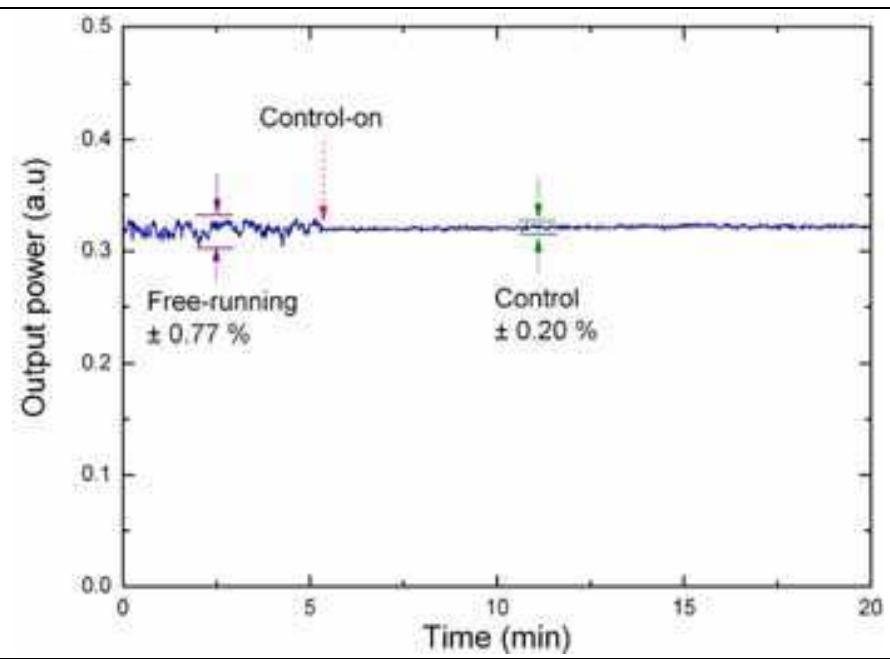

Fig. 5. Long term stability of the Ar+ laser before and after operating the control loop. The a.u means the arbitrary unit.

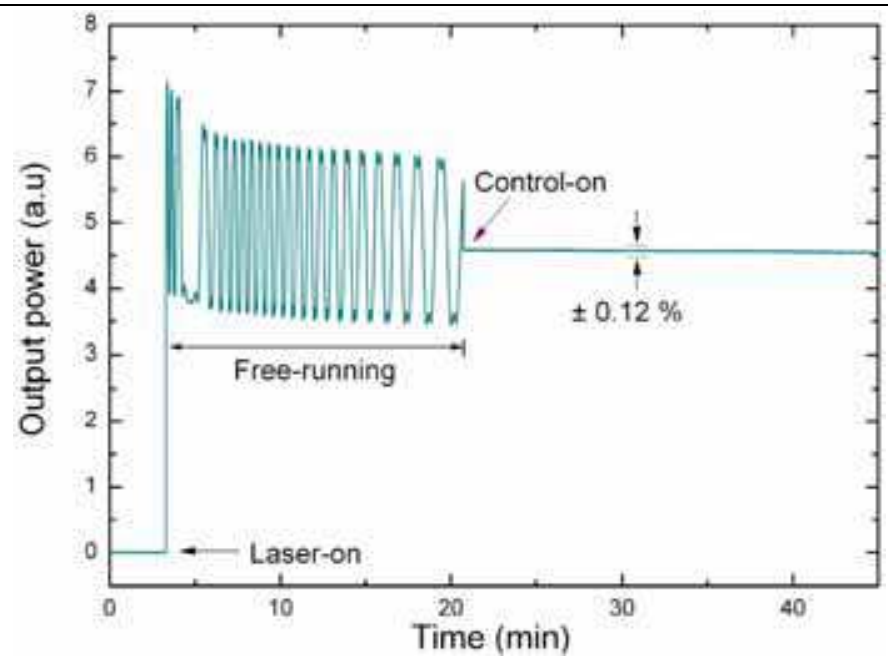

Fig. 6. Long term stability of the internal mirror He-Ne laser.

In addition, we have applied the stabilization part to a He-ne laser, and obtained the \pm 0.12 \% stability as shown in Fig. 6. An important thing is that the laser has always to be linearly polarized. The reason is as follow. First, if non-polarized beam is used, the first order beam power is considerably decreased because the AOM efficiency is maximized when the direction of polarization of the beam has to be perpendicular to the direction of propagation of acoustic wave passing through the AOM crystal. The second, if we look into a laser gain profile with respect to multi longitudinal mode, each mode ha P or S-polarization, so that the oscillating beam is mixed with $\mathrm{P}$ and S-polarization each other. These non-polarization 
beams were not controlled properly. In case of $\mathrm{Ar}+$ laser, the plasma tube ends were cut with Brewster angle. Therefore the extracted beam was linearly polarized, so that we need not use any additional linear polarizer.

\subsection{Beam focusing using the autofocusing technique}

It is important to maintain constantly writing beam focus on the all of a surface during a fabrication for producing good quality of CGH and DOE. A principal factor affecting the variation of the focus is the tilted surface of a substrate. Because of the inclination, the size of the focal point is varied during the fabrication. In the result the line width is broader and the depth is shallower. In order to correct the defocusing amount which arises from the above reason, we have introduced an astigmatic strategy but enhanced new one, one of the active autofocusing methods, and produced an independent autofocusing controller to overcome speed limitation. The mechanism of the autofocusing system is as follows: an auxiliary reflection beam (LD in Fig. 1) from the surface of a substrate goes through a set of cylindrical lenses, and makes various intensity shapes on the focal plane depending on the distance variation between the surface and the cylindrical lens set. Here, the perpendicular cylindrical lens set plays an important role because it change sharply the intensity shape in $x$ or y-direction according to the distance, so it maximizes astigmatism that it can increase defocusing amount and sensitivity. To this end, it is possible to make large scale optical fabrication maintaining uniform precision in comparison with the previous astigmatic method which is only applicable to small scale cases such as CD/DVD pickup. The intensity shape variation is accepted by a quadrant detector (EG\&G UV140BQ-4) and then introduced to a computer by four different cables. The four signals from the quadrant detector (QD) make an error signal in the computer and feedback the error signal to the PZT actuator (PI P-721.0LQ) supported to the micro-objective lens to maintain the constant focal point on the substrate. By doing so, the constant focus can be formed on the surface. However, this method has a speed limitation of $9 \mathrm{~Hz}$ - if a spindle rotates one revolution in one second, then the autofocusing operates one time every 40 degrees - so that it is impossible to control the autofocusing on the high speed rotation with more than the angular velocity of $360 \mathrm{o}$. To improve the limitation, we have made an autofocusing controller built-in an electronic circuitry independent of the computer. The details of our system are explained as two following subsections.

Depending on the LD beam shape reflected from a target surface through an optical system, QD makes different photocurrents at four detecting areas for the autofocusing. Figure 7 shows the optical configuration of the autofocusing part. Firstly, we attached a band-pass filter, a linear polarizer, a biconvex lens, and a cube beam splitter to the writing head. The role of each part is as follows: the band-pass filter passes only $635 \mathrm{~nm}$ LD beam except for the $488 \mathrm{~nm}$ and $457.9 \mathrm{~nm}$ writing beam. The linear polarizer plays a role of reducing the residue reflection beam in the writing head that acts as a noise source on QD. The biconvex lens adjusts suitably beam scaling on QD and the optical distance from the target surface to $\mathrm{QD}$, and the cube beam splitter reflects off most LD beam (about $90 \%$ ) coming from the surface to the cylindrical lens assembly. The other beam from the beam splitter (about $10 \%$ ) is monitored by a Charge Coupled Device (CCD). Secondly, we installed the cylindrical lens assembly composed of two cylindrical lenses right angle to each other on the linear stage. Lastly, QD mounted on a XYZ translator was also set up on the stage. In Fig. 7, the beam reflected from the material surface goes though the series of optics and makes a particular spot pattern near the focal planes. The QD is placed between two astigmatic foci that two 
cylindrical lenses originally have, namely at the location where the intensity pattern looks to be a perfect circle as shown in Fig. 7 (position 2). The orientation of QD lines should be $45^{\circ}$ or $1355^{\circ}$ with respect to the tangential plane of the cylindrical lenses. If the distance between the material surface and the objective is changed, the intensity patterns on QD will also be changed, thereby bringing about the error signal change. The radius of curvature of each cylindrical lens was $15.5 \mathrm{~mm}$, the focal length was $30 \mathrm{~mm}$, the space between two lenses was $5.7 \mathrm{~mm}$, and the effective focal length of two lenses was $15.7 \mathrm{~mm}$.

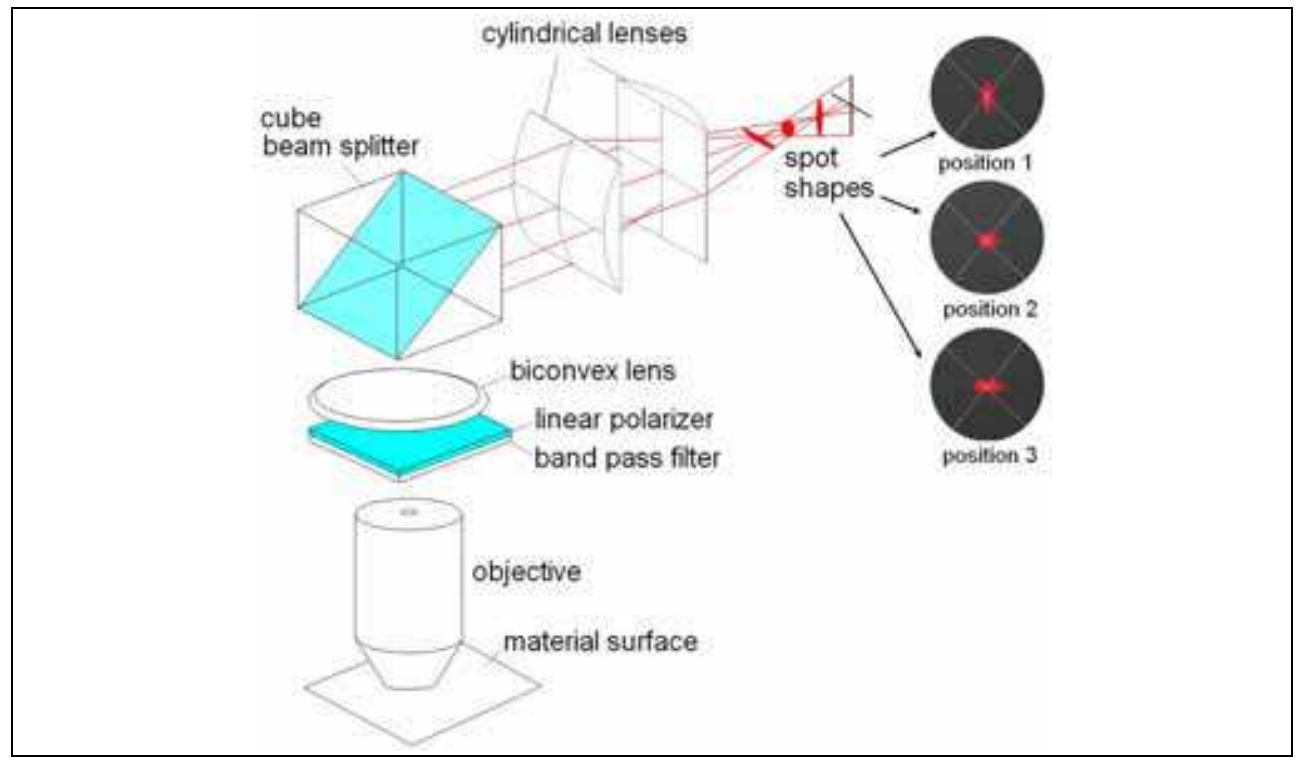

Fig. 7. Optical components to generate the auto-focusing error signal. The three different kinds of spot shape are formed on the QD according to the distance between the objective and the material surface. The position 2 indicates the exact focal point.

Our autofocusing controller is possible to achieve the high speed control up to $150 \mathrm{~Hz}$ (PZT modulation limit). Each signal received by QD is guided into the controller through four different BNC cables. In the controller, first, the guided current signals are converted into the voltage signals by current to voltage converters. In this process, the capacitance of a condenser affect considerably to the response time of the PZT actuator. Figure 8 shows Bode diagram according to the capacitance change in which the reducer the capacitance, the faster the actuator response. The optimal capacitance we found here is $1 \mathrm{nF}$ as shown in Fig. 8(c). When the capacitance was less than this, the actuator was overshot even to the minimum gain in our experiment. Especially if no condenser was used like in Fig. 8(d), a lot of noise was occurred in the process of current to voltage conversion. Under the larger capacitance such as Fig. 8(a), the autofocusing speed could not catch up with the rotation speed of 360 o/s. Next, the converted voltage signals undergo a series of calculation (addition and subtraction), then makes a normalized error signal $\mathrm{e}_{\mathrm{N}}(\mathrm{I})$ as shown in the following Eq. (1).

$$
e_{N}(I)=\frac{I_{a}+I_{d}-\left(I_{b}+I_{c}\right)}{I_{a}+I_{d}+\left(I_{b}+I_{c}\right)}
$$




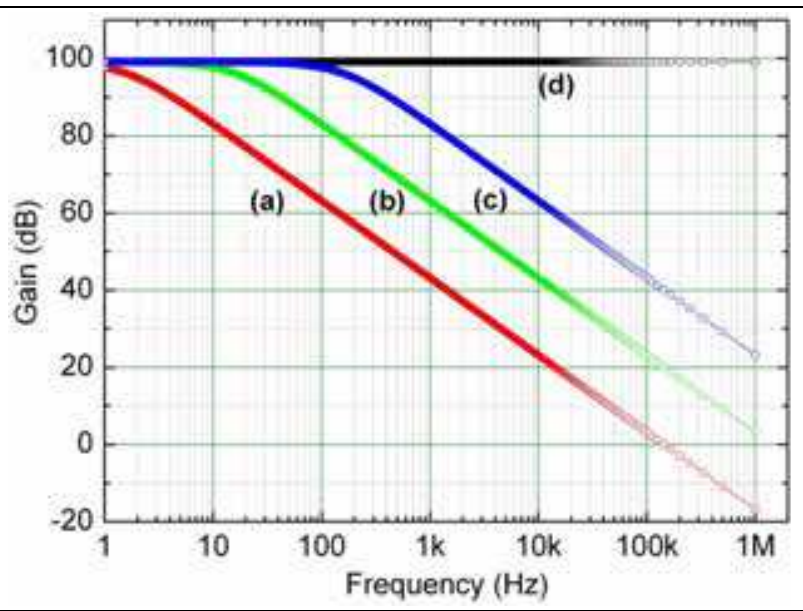

Fig. 8. Bode diagram about current to voltage conversion. (a) and (b) are the capacitance of $100 \mathrm{nF}$ and $10 \mathrm{nF}$. (c) is in the case of optimal capacitance of $1 \mathrm{nF}$ for fast response of the PZT actuator. (d) shows no condenser case.

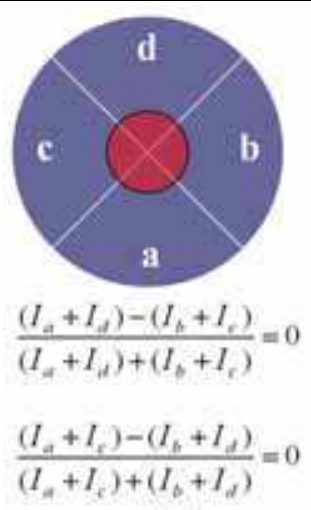

(a)

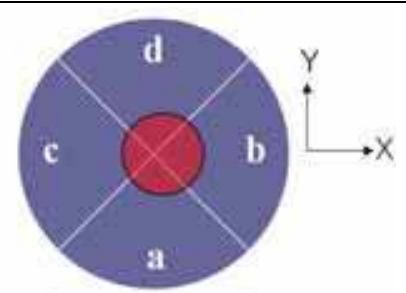

$\frac{\left(I_{a}+I_{d}\right)-\left(I_{b}+I_{f}\right)}{\left(I_{a}+I_{d}\right)+\left(I_{b}+I_{e}\right)}=0$

$\frac{\left(I_{a}+I_{c}\right)-\left(I_{b}+I_{d}\right)}{\left(I_{b}+I_{c}\right)+\left(I_{b}+I_{b}\right)} \neq 0$

(b)

Fig. 9. Alignment of QD to find out center at the exact focal point. (a) The exact QD center, (b) a case of off-center.

where a, b, c, and d indicate the four sections of QD as shown in the Fig. 9(b), respectively. This normalization is able to reduce noise arising from the sudden light intensity fluctuation caused by dusts and/or stains on the surface of the substrate. In other words, this sudden intensity variation degrades the autofocusing function because of acting as a noise source. The normalized error $\mathrm{e}_{\mathrm{N}}(\mathrm{I})$ is divided into three parts and applied PID (Proportional Integral Derivative) control to each part. Multiplying suitable gains and summing each part, then a final focusing error signal (FES) is made. It can be written as

$$
F E S=K_{p} \times e_{N}(I)+K_{i} \times \int e_{N}(I) d I+K_{d} \times \frac{d e_{N}(I)}{d I},
$$


where $K_{p}, K_{i}$, and $K_{d}$ are the proportional, integral, and derivative gain, respectively. We have gotten better result than the case in which the proportional gain was only given. By doing so, residual error after the autofocusing was reduced more (less than $1 \mu \mathrm{m}$ ) and besides the control range was extended twice from $\pm 25 \mu \mathrm{m}$ to $\pm 50 \mu \mathrm{m}$. Now, the remainder of work is to locate the laser beam shape to the center of QD. One problem is that when we look for the zero point for the autofocusing, the error signal would be zero even in the case of Fig. 9(b). To correct this we added a circuit which calculates position error signal (PES) to the controller. PES is given by

$$
\begin{gathered}
e_{N P}(I)=\frac{I_{a}+I_{c}-\left(I_{b}+I_{d}\right)}{I_{a}+I_{c}+\left(I_{b}+I_{d}\right)}, \\
P E S=K_{p} \times e_{N P}(I),
\end{gathered}
$$

where $e_{N P}(I)$ represents a normalized error signal for the estimation of position error deviated from QD center and $\mathrm{K}_{\mathrm{p}}$ is the proportional gain. By setting FES and PES to be zero, respectively, we can improve the accuracy of our autofocusing control. To find the zero point, QD position with respect to $X$ and $Y$ axes in Fig. 9 was tuned FES and PES to be 0.000 $\pm 0.001 \mathrm{~V}$ at the initial focal point, and then we turned the control switch on with suitable gains $\left(\mathrm{K}_{\mathrm{p}}, \mathrm{K}_{\mathrm{i}}\right.$, and $\mathrm{K}_{\mathrm{d}}$, respectively). The autofocusing controller gives the error signal to PZT actuator to keep the focusing position during the fabrication.

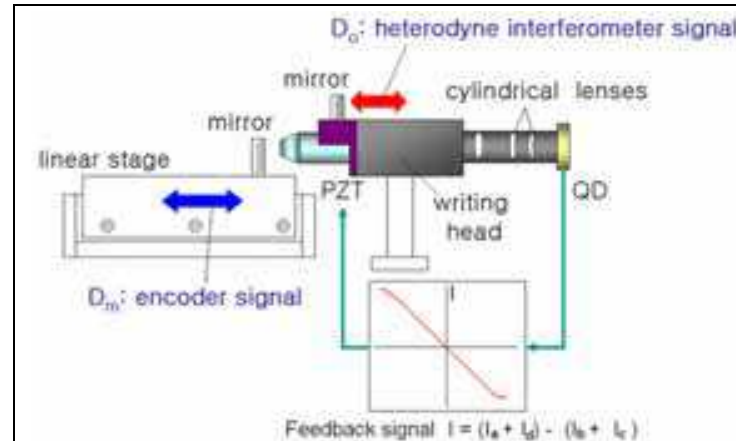

(a)

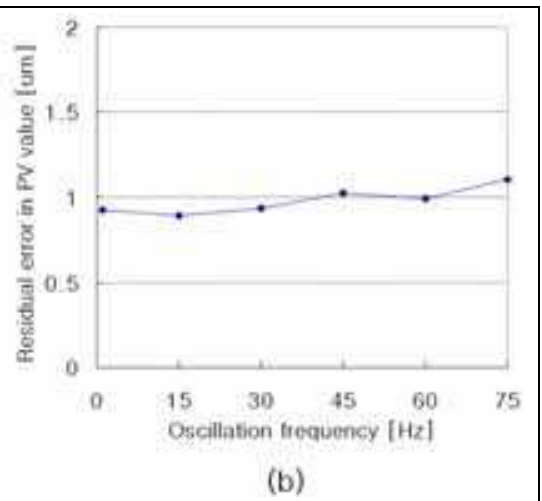

(b)

Fig. 10. (a) The experimental setup to check the performance of the autofocusing system, and (b) the residual error after the autofocusing. The higher frequency (larger than $75 \mathrm{~Hz}$ ) test is meaningless because it is over the Nyquist sampling limitation.

Figure 10 (a) shows a setup to measure the autofocusing error. The mirror attached on the linear stage oscillated, and we measured $D_{m}$ (the mirror movement) and $D_{o}$ (the objective movement) at the same time. After whole test, the autofocusing error $D_{m}-D_{o}$ was less than $1.1 \mu \mathrm{m}$ in peak-to-valley (PV) value as shown in Fig. $10(\mathrm{~b})$. When the focusing point moved $1 \mu \mathrm{m}$, the line width change of the pattern was approximately $3.6 \%$ with 100X objective (NA: 0.7, depth of focus: $0.6 \mu \mathrm{m}$ ). Therefore we suppose that the 1- $\mu \mathrm{m}$ focusing error is allowable.

Figure 11 shows CCD (charge coupled device) snap shots showing the focusing variation on the rotating surface. As shown in Fig. 11(a), the variation of the focus, due to surface tilting 
(the greatest contribution to the focus variation), according to the rotation angle can be observed. The focus size at $0 \circ$ is increasing as the substrate rotates, and then back to normal after one rotation. On the contrary, however, once we apply the autofocusing control to it, the focus size is nearly invariant for one rotation. To confirm system performance in detail, we carried out writing tests divided into two parts, that is to say, with and without the autofocusing control. The target glass wafer is coated with chromium of $100 \mathrm{~nm}$ thickness. The test writing results given the surface radius change from $4.6 \mathrm{~mm}$ to $14 \mathrm{~mm}$ and $10 \mu \mathrm{m}$ line to line spacing is shown in Fig. 12. As in the inner area of Fig. 12(a), when turn the autofocusing off, the fabricated pattern in the region out of the focus is entirely missed, and what is more, the written areas are also dimming even if the focusing region is little deviated from the focus. Applying the autofocusing control to the whole surface, on the

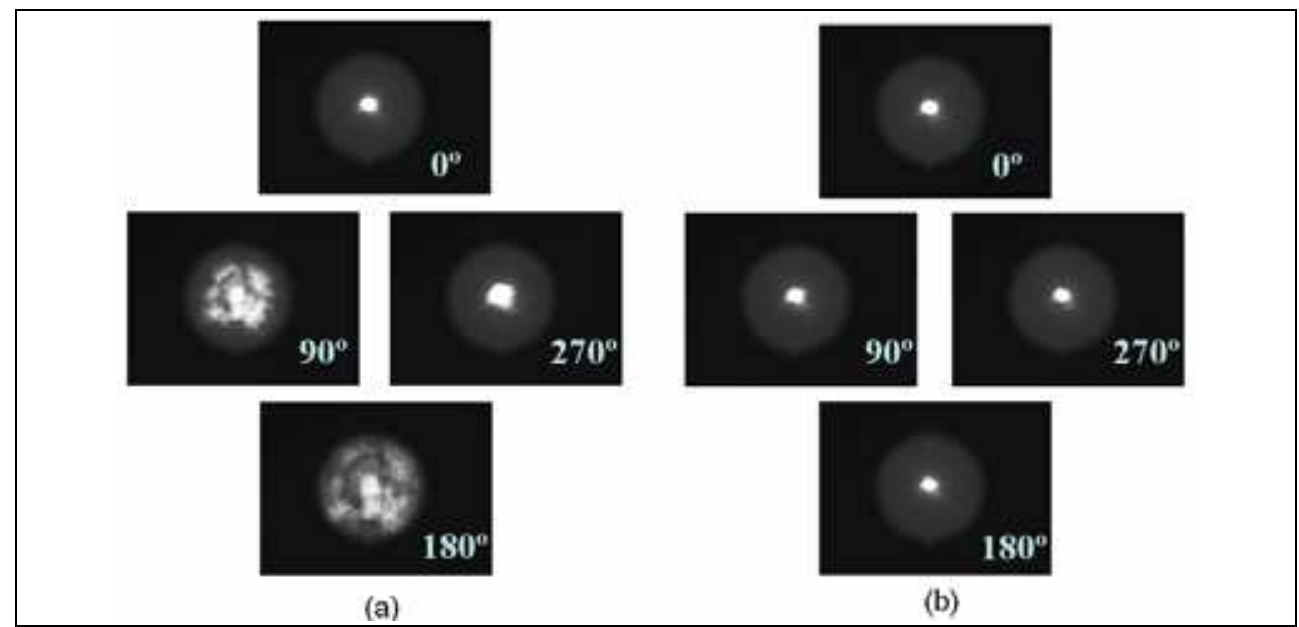

Fig. 11. CCD snap shots of a focal point variation on the rotating substrate (a) before and (b) after the auto-focusing control.

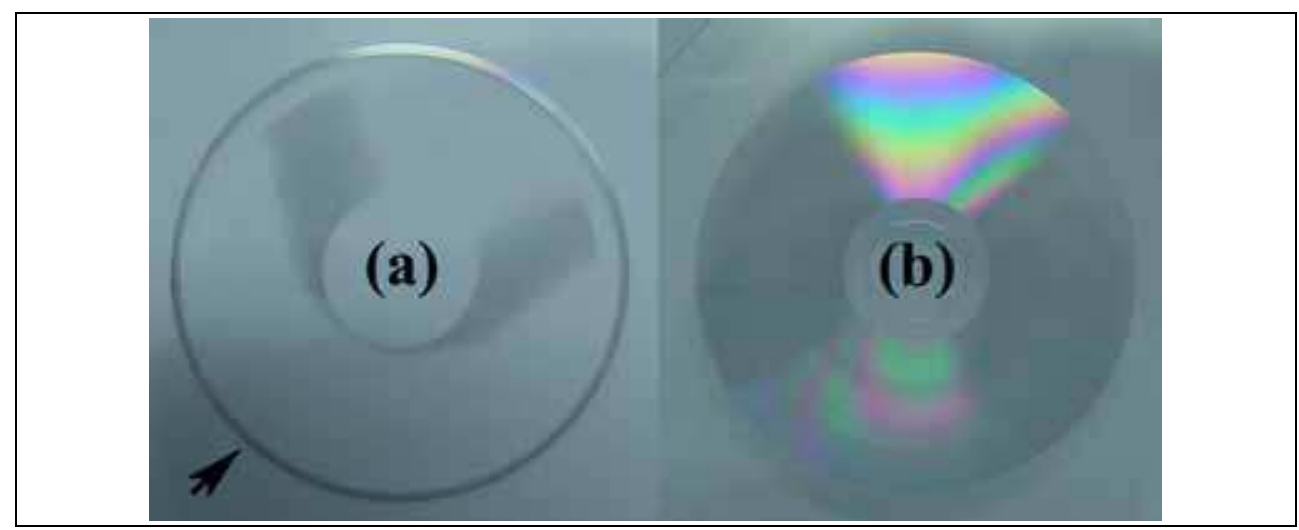

Fig. 12. Fabrication results: comparison of (a) before with (b) after the auto-focusing control. The arrowed circles in (a) is a result with the auto-focusing control to show the significance of it. 
other hand, uniform patterns are well written as shown in the outermost arrowed circle in Fig. 12(a). Even in a small area there is a big difference whether the autofocusing is present or not, to say nothing of a large one. From the comparative results, the significance of autofocusing is emphasized here. Figure 12(b) shows that the patterns on the surface are successfully fabricated to the whole area by means of the autofocusing control. We could also confirm the uniform linewidth of $2.0 \mu \mathrm{m}$ as shown in Fig. 13 using a commercial whitelight scanning interferometer. In addition, we have accomplished the linewidth of $0.6 \mu \mathrm{m}$ by means of controlling the source laser power. In the light of these facts, we can assure that our autofocusing system is well operated.

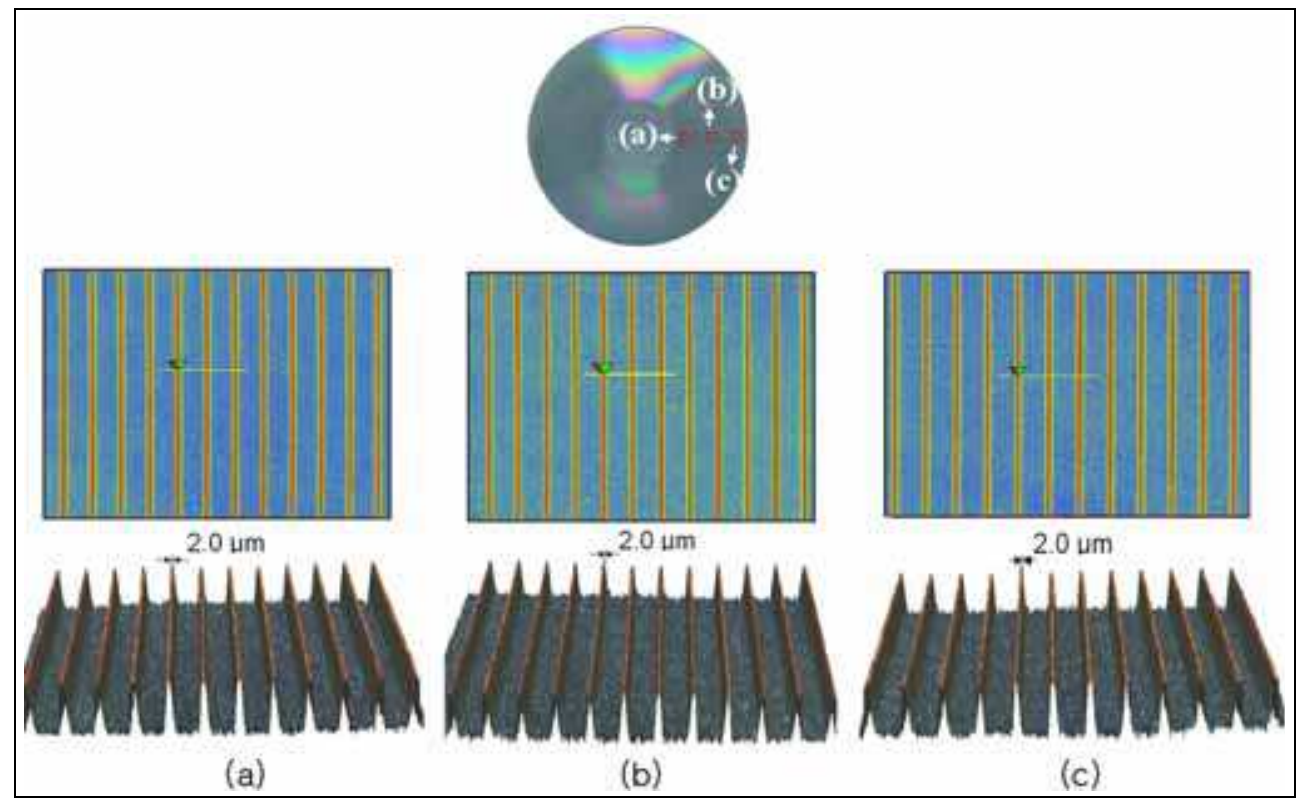

Fig. 13. Fabrication results obtained by a commercial white-light scanning interferometer (field of view: $124 \mu \mathrm{m} \times 93 \mu \mathrm{m}$, magnification: 50X). Region (a), (b), and (c) show uniform linewidth of $2.0 \mu \mathrm{m}$, respectively.

\section{Applications}

\subsection{Computer Generated Hologram (CGH)}

Figure 14 (a) show a typical CGH fabricated by the direct laser lithographic system. The root-mean-square wavefront error of the $\mathrm{CGH}$ was $0.03 \lambda(\lambda$ means the wavelength of the He-Ne laser, $632.8 \mathrm{~nm}$.) as shown in Fig. 15.

When we fabricate a CGHm the center alignment of the writing head is an important error source. To align the center (origin) precisely, we used the tilt table and the $Y$ stage as shown in Fig. 1.

We used a new alignment method by using four spirals. The procedure is here: (a) Fabricating the first spiral on the sample. (b) Rotating the sample $90^{\circ}$ using the rotary stage, and then fabricating the second spiral. (c) Rotating the sample $90^{\circ}$ again, and fabricating the 
third spiral. (d) Rotating the sample 90\%, and fabricating the last spiral. If the center alignment is good enough, the spiral pattern looks like Fig. 16 (a). In this figure, the amount of misalignment was $168 \mathrm{~nm}$. This number was calculated by the least square line fitting.

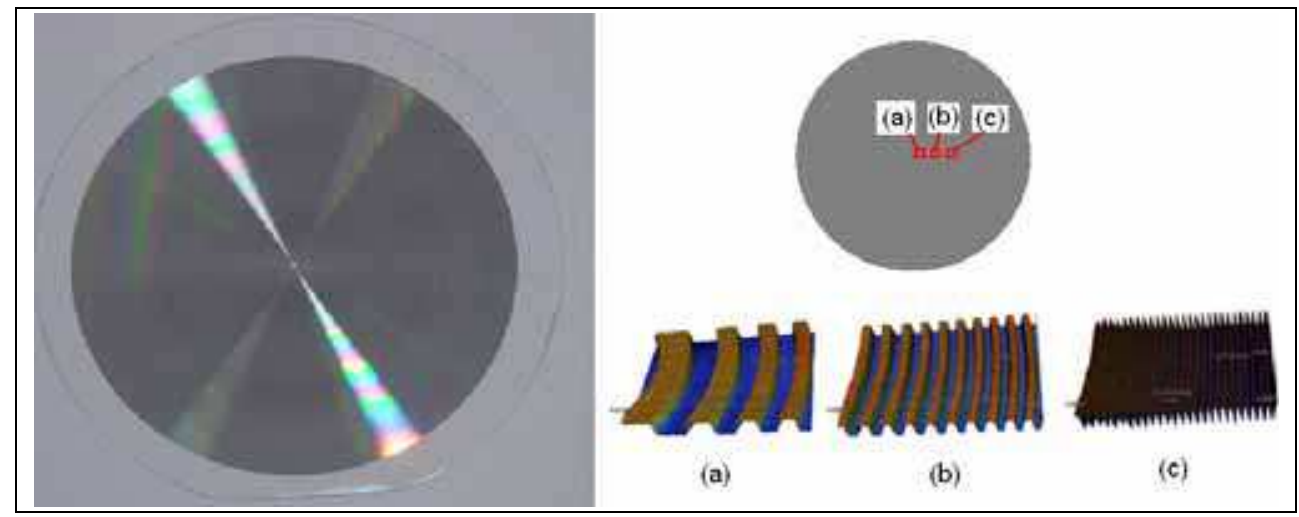

Fig. 14. Typical CGH we fabricated. The fabrication results measured by the white-light scanning interferometer (field of view: $124 \mu \mathrm{m} \times 93 \mu \mathrm{m}$, magnification: 50X).

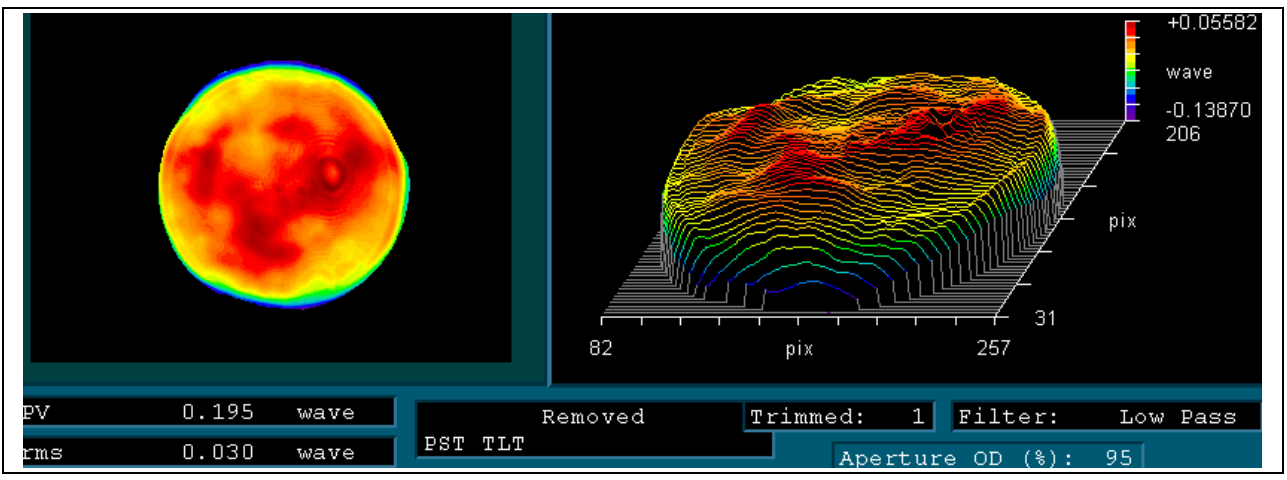

Fig. 15. Wavefront error of the CGH measured by a commercial Fizeau interferometer.

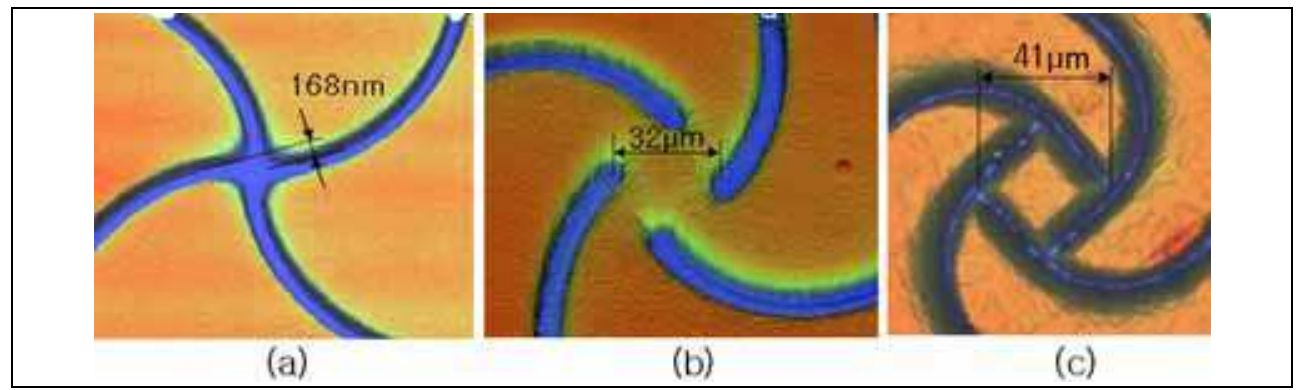

Fig. 16. Four spiral patterns: (a) good alignment case, (b) plus-direction misalignment case, and (c) minus-direction misalignment case. 


\subsection{Reference chromium patterns on a silicon wafer}

In this section, we describe the second application of the direct laser lithographic system. In recent years, the semi-conductor industry has required a new inspection method for internal defects of the silicon wafer. To effectively find these small defects, some companies are developing new inspection equipment using the infrared light source. The infrared beam usually penetrates the silicon, and is scattered on the defect. Using this phenomenon the inspection equipment is able to find out the precise position and the size of each defect in the silicon wafer. At this time the precision of the equipment mainly depends on the coordinate system of the equipment itself. Therefore the equipment should be calibrated before inspection by using a well-made reference specimen that has two-dimensional array patterns whose xy-coordinates are already known. A typical fabrication procedure of the reference wafer is as follows: (a) preparing a wafer that has no internal defect, (b) polishing both sides of the wafer, (c) coating the chromium on the top side of the wafer, (d) patterning on the coated side, and (e) etching the wafer. In this procedure, chromium is preferable to other materials because it can effectually block the infrared beam with a relatively thin thickness. The other advantage of chromium is its easiness for fabrication.
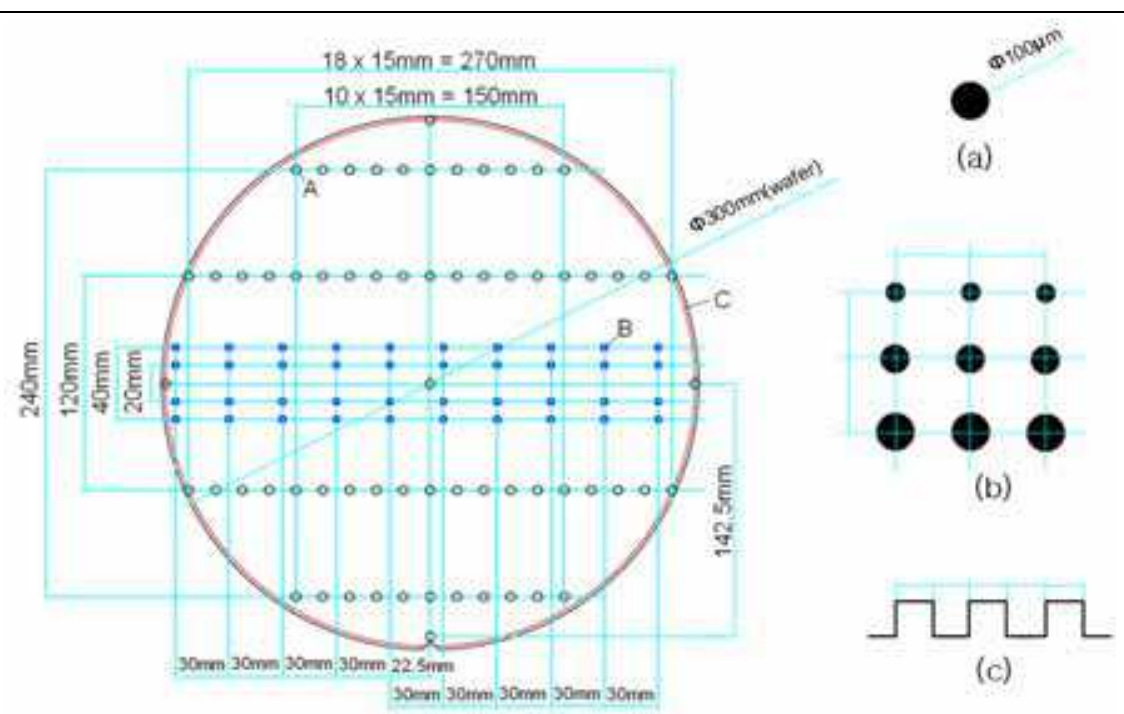

Fig. 17. 300-mm-diameter reference wafer. Using pattern C, the equipment can automatically level the reference wafer.

In spite of the advantages of the chromium, there are two problems. First, the semi-conductor industry requires a maximum 300-mm wafer as illustrated in Fig. 17. With the well-known Ebeam method, however, it is hard to achieve this size. Therefore we applied the direct laser lithography technique instead of the e-beam. The second problem is that the most effective chromium etchant (we used the etchant consisting of six parts of $25 \%$ solution of $\mathrm{K}_{3} \mathrm{Fe}(\mathrm{CN})_{6}$ and one part of $25 \%$ solution of $\mathrm{NaOH}$.) seriously erodes the silicon. To prevent this, we propose a new method using a $\mathrm{SiO}_{2}$ layer whose thickness is from $100 \mathrm{~nm}$ to $200 \mathrm{~nm}$. This layer can protect the silicon wafer from the etchant, and does not disturb the measurement since the infrared beam penetrates the $\mathrm{SiO}_{2}$ laser. The details are described in Fig. 18. 


\begin{tabular}{|c|c|c|c|c|}
\hline & $\mathrm{SiO}_{2}(\sim 100 \mathrm{~nm})$ & $\mathrm{Cr}(\sim 70 \mathrm{~nm})$ & & $\begin{array}{l}\text { etching with } \\
\mathrm{K}_{3} \mathrm{Fe}(\mathrm{CN})_{6} \\
+\mathrm{NaOH}\end{array}$ \\
\hline Silicon wafer & Silicon wafer & Silicon wafer & Silicon wafer & Silicon wafer \\
\hline
\end{tabular}

Fig. 18. Proposed procedure to fabricate the reference wafer

Figures 19, 20 and 21 show the fabrication result of each pattern. The thicknesses of the patterns are nearly $70 \mathrm{~nm}$, which is enough to block the infrared light as shown in Fig. 19 (a) and 20 (a).

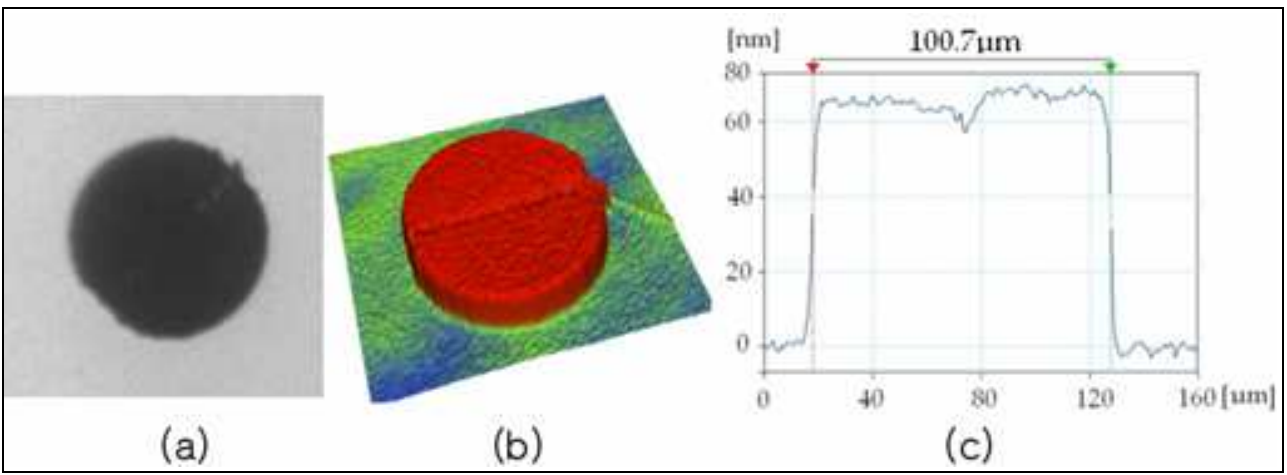

Fig. 19. Pattern A: (a) infrared microscopic view, (b) three-dimensional shape measured by a white-light scanning interferometer, and (c) a section profile. The measured diameter of the pattern was $100.7 \mu \mathrm{m}$ instead of $100.0 \mu \mathrm{m}$. We supposed that this deviation is mainly caused by the fluctuation of the intensity and the focus point. It is also affected by the etching time.

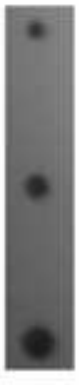

(a)

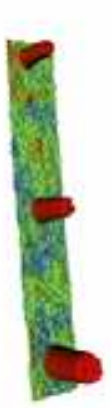

(b)

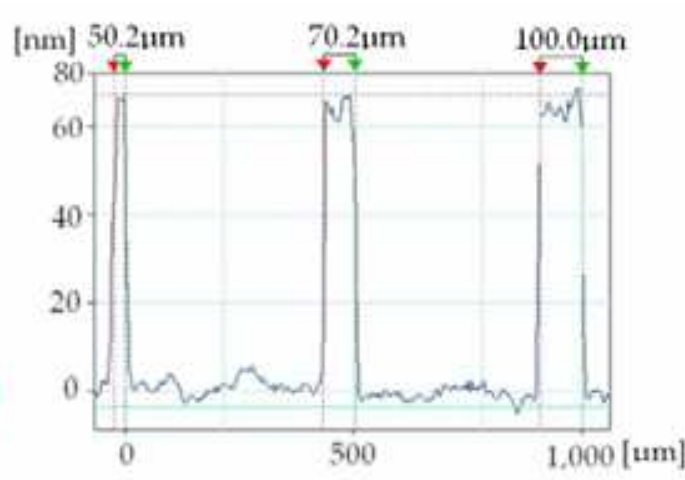

(c)

Fig. 20. A column of pattern B: (a) infrared microscopic view, (b) three-dimensional shape measured by a commercial white-light scanning interferometer, and (c) a section profile. 


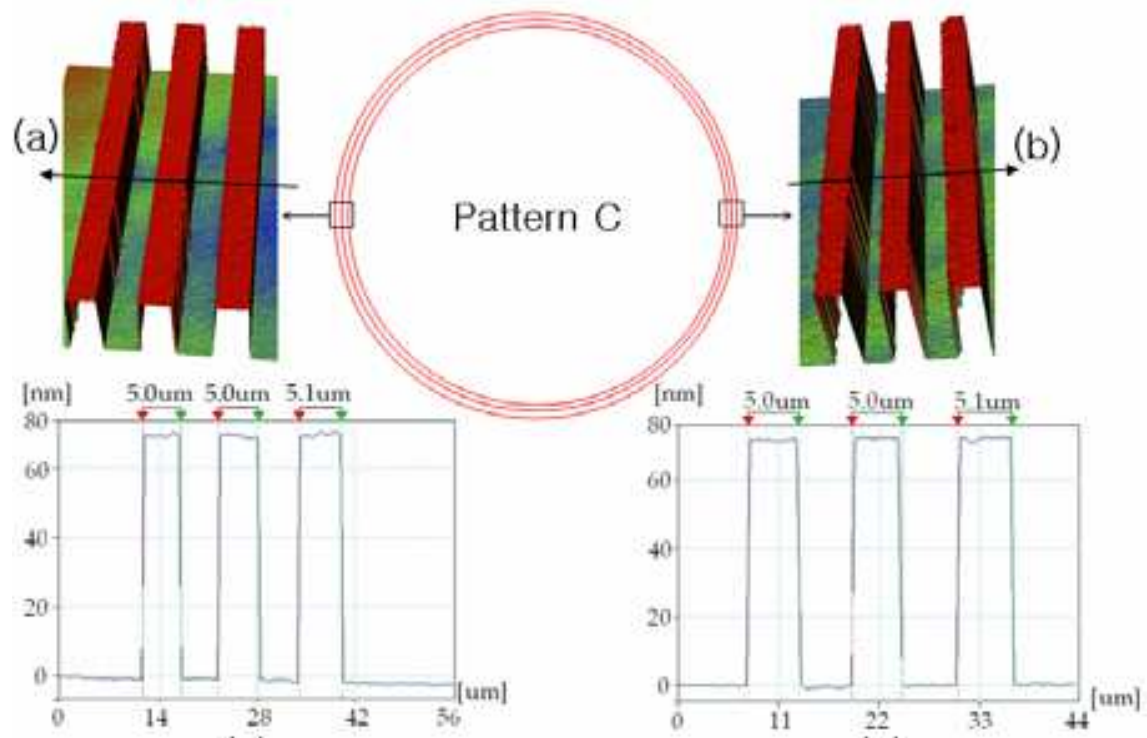

(a)

(b)

Fig. 21. Pattern C.

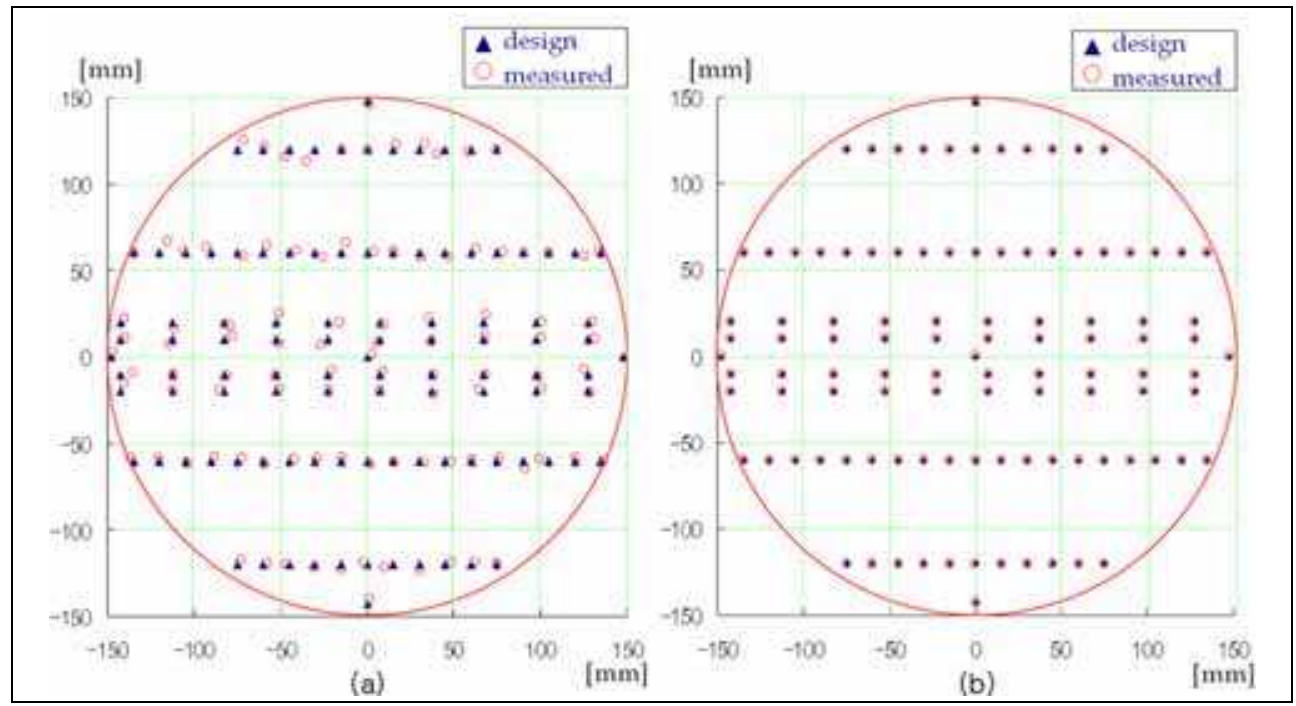

Fig. 22. Systematic error of the infrared inspection equipment (a) before and (b) after calibration. The triangular mark represents the designed position (see Fig. 17), while the circular mark means the measuring result. The positions of the circular marks are intentionally exaggerated.

Using the reference wafer, we finally tested the inspection equipment. The circular marks shown in Fig. 22 (a) represent the systematic error of the inspection equipment, in which the 
absolute values of the maximum error were measured as $8.9 \mu \mathrm{m}$ for horizontal- and $12.6 \mu \mathrm{m}$ for vertical-direction. We calibrated the equipment with the reference wafer, and successfully reduced the systematic error of the equipment as shown in Fig. 22 (b). After calibration, the maximum errors were $0.7 \mathrm{~nm}$ for horizontal- and $0.9 \mathrm{~nm}$ for verticaldirection in absolute value.

\section{Conclusion}

The direct laser lithography is a useful technique to fabricate a large precision patterns such as CGHs, DOEs, and reference wafers. The typycal lithograpic system we have built can write up to $360-\mathrm{mm}$ diameter substrate coated with chromium or photoresist film. The writing source were stabilized by using the AOM, the PD, and the servo controller. We also achieve the high speed and large range autofocusing system using two cylindrical lenses. Then we fabricated various CGH, zone plates, and the 300-reference wafer . 150-mm, 200$\mathrm{mm}$, reference wafers were also successfully fabricated using our system.

\section{References}

Offner, A. \& Malacara, D. (1992). Optical Shop Testing 2nd edition, Wiley, 0-471-52232-5, New York

Poleshchuk, A. G.; Churin, E. G.; Koronkevich, V. P.; Korolkov, V. P.; Kharussov, A. A.; Cherkashin, V. V.; Kiryanov, V. P.; Kiryanov, A. V.; Kokarev, S. A. \& Verhoglyad, A. G. (1999). Polar coordinate laser pattern generator for fabrication of diffractive optical elements with arbitrary structure, Appl. Opt., Vol. 38, No. 8, 1295-1301, 00036935

Asfour, J. \& Poleshchuk, A. G. (2006). Asphere testing with a Fizeau interferometer based on a combined computer-generated hologram, J. Opt. Soc. Am. A, Vol. 23, No. 1, 172$178,1084-7529$

Ogata, S.; Tada, M. \& Yoneda, M. (1994). Electron-beam writing system and its application to large and high-density diffractive optic elements, Appl. Opt., Vol. 33, No. 10, 2032-2038, 0003-6935

Kim, D.; Rhee H.; Song, J. \& Lee, Y. (2007). Laser output stabilization for direct laser writing system by using an acousto-optic modulator, Rev. Sci. Instrum., Vol. 78, No. 10, 103110/1-103110/4, 0034-6748

Rhee H.; Kim, D. \& Lee, Y. (2008). 300-mm reference wafer fabrication by using direct laser lithography, Rev. Sci. Instrum., Vol. 79, No. 10, 103103/1-103103/5, 0034-6748

Rhee H.; Kim, D. \& Lee, Y. (2009). Realization and performance evaluation of high speed autofocusing for direct laser lithography, Rev. Sci. Instrum., Vol. 80, No. 7, 073103/1-073103/5, 0034-6748

Goodman, J. W. (1996). Introduction to Fourier optics 2nd edition, McGraw-Hill, 0-07-114257-6, Singapore

Deck L. \& deGroot P. (1994). High-speed noncontact profiler based on scanning white-light interferometer, Appl. Opt., Vol. 33, No. 31, 7334-7338, 0003-6935

Ye, J.; Takac, M.; Berglund, C. N.; Owen, G. \& Pease, R. F. (1997). An exact algorithm for selfcalibration of two-dimensional precision metrology stages, Prec. Eng., Vol. 20, No. $1,16-32,0141-6359$ 


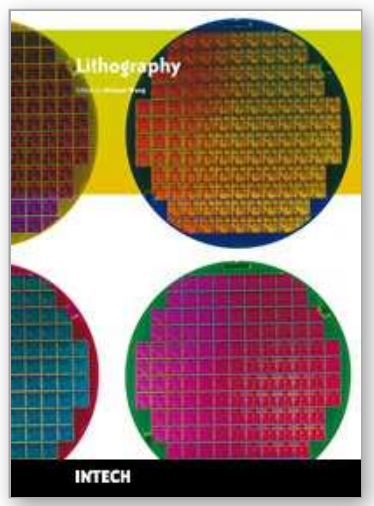

\author{
Lithography \\ Edited by Michael Wang
}

ISBN 978-953-307-064-3

Hard cover, 656 pages

Publisher InTech

Published online 01, February, 2010

Published in print edition February, 2010

Lithography, the fundamental fabrication process of semiconductor devices, plays a critical role in micro- and nano-fabrications and the revolution in high density integrated circuits. This book is the result of inspirations and contributions from many researchers worldwide. Although the inclusion of the book chapters may not be a complete representation of all lithographic arts, it does represent a good collection of contributions in this field. We hope readers will enjoy reading the book as much as we have enjoyed bringing it together. We would like to thank all contributors and authors of this book.

\title{
How to reference
}

In order to correctly reference this scholarly work, feel free to copy and paste the following:

Hyug-Gyo Rhee (2010). Direct Laser Lithography and Its Applications, Lithography, Michael Wang (Ed.), ISBN: 978-953-307-064-3, InTech, Available from: http://www.intechopen.com/books/lithography/direct-laserlithography-and-its-applications

\section{INTECH}

open science | open minds

\section{InTech Europe}

University Campus STeP Ri

Slavka Krautzeka 83/A

51000 Rijeka, Croatia

Phone: +385 (51) 770447

Fax: +385 (51) 686166

www.intechopen.com

\section{InTech China}

Unit 405, Office Block, Hotel Equatorial Shanghai

No.65, Yan An Road (West), Shanghai, 200040, China

中国上海市延安西路65号上海国际贵都大饭店办公楼405单元

Phone: +86-21-62489820

Fax: +86-21-62489821 
(C) 2010 The Author(s). Licensee IntechOpen. This chapter is distributed under the terms of the Creative Commons Attribution-NonCommercialShareAlike-3.0 License, which permits use, distribution and reproduction for non-commercial purposes, provided the original is properly cited and derivative works building on this content are distributed under the same license. 